\title{
A Quasistatic Contact Problem for Viscoelastic Materials with Slip-Dependent Friction and Time Delay
}

\author{
Si-sheng Yao ${ }^{1,2}$ and Nan-jing Huang ${ }^{1}$ \\ ${ }^{1}$ Department of Mathematics, Sichuan University, Chengdu 610064, China \\ ${ }^{2}$ Department of Mathematics, Kunming University, Kunming 650221, China \\ Correspondence should be addressed to Nan-jing Huang, nanjinghuang@hotmail.com
}

Received 27 June 2012; Revised 26 September 2012; Accepted 16 November 2012

Academic Editor: Marco Paggi

Copyright (C) 2012 S.-s. Yao and N.-j. Huang. This is an open access article distributed under the Creative Commons Attribution License, which permits unrestricted use, distribution, and reproduction in any medium, provided the original work is properly cited.

\begin{abstract}
A mathematical model which describes an explicit time-dependent quasistatic frictional contact problem between a deformable body and a foundation is introduced and studied, in which the contact is bilateral, the friction is modeled with Tresca's friction law with the friction bound depending on the total slip, and the behavior of the material is described with a viscoelastic constitutive law with time delay. The variational formulation of the mathematical model is given as a quasistatic integro-differential variational inequality system. Based on arguments of the timedependent variational inequality and Banach's fixed point theorem, an existence and uniqueness of the solution for the quasistatic integro-differential variational inequality system is proved under some suitable conditions. Furthermore, the behavior of the solution with respect to perturbations of time-delay term is considered and a convergence result is also given.
\end{abstract}

\section{Introduction}

The phenomena of contact between deformable bodies or between deformable and rigid bodies are abound in industry and daily life. Contact of braking pads with wheels and that of tires with roads are just a few simple examples [1]. Because of the importance of contact processes in structural and mechanical systems, a considerable effort has been made in their modeling and numerical simulations (see [1-4] and the references therein). What is worth to be taken particularly is some engineering papers that discussed the developed mathematical modeling to a practically interesting problem $[5,6]$. Owing to their inherent complexity, contact phenomena are modeled by nonlinear evolutionary problems that are difficult to analyze (see [1]). The first work concerned with the study of frictional contact problems within the framework of variational inequalities was made in [7]. Comprehensive 
references for the analysis and numerical approximation of contact problems include $[1,8]$. Mathematical, mechanical, and numerical state-of-the-art on contact mechanics can be found in the proceedings $[9,10]$ and in the special issue [11].

When a viscoelastic material undergoes a small deformation gradient with a relatively slow force applied, the process of the relative contact problem can be modeled by a quasistatic system. At a relatively short duration, the effect of temperature changes caused by energy dissipation on the deformation of the material is usually negligible. Rigorous mathematical treatment of quasistatic problems is a test of recent years. The reason lies in the considerable difficulties that the process of frictional contact presents in the modeling and analysis because of the complicated surface phenomena involved (see [12]). By employing Banach's fixed point theorem, Chau et al. [13] got some existence and uniqueness results for two quasistatic problems which describe the frictional contact between a deformable body and an obstacle. They also proved that the solution of the viscoelastic problem converges to the solution of the corresponding elastic problem. By using arguments for time-dependent elliptic variational inequalities and Banach's fixed point theorem, Rodriguez-Aros et al. [10] dealt with the existence of a unique solution to an evolutionary variational inequality with Volterra-type integral term. Delost and Fabre [14] presented a valid approximation method for a quasistatic abstract variational inequality with time-independent constraint and applied its results to the approximation of the quasistatic evolution of an elastic body in bilateral contact with a rigid foundation. Very recently, Vollebregt and Schuttelaars [15] studied the quasistatic analysis of a contact problem with slip-velocity-dependent friction. For more works concerned with the quasistatic contact problems, we refer to $[1,16]$ and the references therein.

Quasistatic contact problems for viscoelastic or other materials with explicit timedependent operators were investigated in a large number of papers. Applying the theory of evolutionary hemivariational inequality, Migórski et al. [17] proved the existence and the regularity properties of the unique weak solution to a nonlinear explicit time-dependent elastic-viscoplastic frictional contact problem with multivalued subdifferential boundary conditions. In [18], Migórski et al. considered a class of quasistatic contact problems for explicit time-dependent viscoelastic materials with subdifferential frictional contact conditions. Based on the fixed point theorem for multivalued mappings and variationalhemivariational inequality theory, Costea and Matei [19] proved the existence of weak solution for the general and unified framework contact models. They also discussed the uniqueness, the boundedness, and the stability of the weak solution under some suitable conditions.

It is well known that time-delay phenomena are frequently encountered in various technical systems, such as electric, pneumatic and hydraulic networks, and chemical processes. For example, regarding polymer under the action of alternative stress, the stress will lag behind the strain, which is just a time delay phenomenon. It gives us a mechanism of the time-delay phenomena appeared in contact problems. Comincioli [20] proved the existence and uniqueness for a kind of variational inequality with time-delay. For general results of variational inequalities with time delay, we refer to [21-23]. However, to the best of our knowledge, there is no papers to study contact problems for viscoelastic materials with time-delay.

Motivated and inspired by the work mentioned above, in this paper, we introduce and study a mathematical model which describes an explicit time-dependent quasistatic frictional contact problem between a deformable body and a foundation, in which the contact is bilateral, the friction is modeled with Tresca's friction law with the friction bound depending on the total slip, and the behavior of the material is described with a viscoelastic constitutive 
law with time delay. We give the variational formulation of the mathematical model as a quasistatic integro-differential variational inequality system. By using the arguments of timedependent variational inequality and Banach's fixed point theorem, we prove an existence and uniqueness of the solution for the quasistatic integro-differential variational inequality system under some suitable conditions. Furthermore, we consider the behavior of the solution with respect to perturbations of time-delay term and show a convergence result. The results presented in this paper generalize and improve some known results of $[1,24]$.

The paper is structured as follows. In Section 2, we list the necessary assumptions on the data and derive the variational formulation for the problem. In this part, an example which is assumed to the Kelvin-Voigt viscoelastic constitutive law with long memory is given, which represents a constitutive equation of the form (2.19). In Section 3, we prove the existence and uniqueness of the solution to the quasistatic integro-differential variational inequality system. In Section 4, we study the behavior of the solution with respect to perturbations of time-delay term and derive the convergence result.

\section{Preliminaries}

Let $\mathcal{R}^{d}$ be a $d$-dimensional Euclidean space and $\mathcal{S}^{d}$ the space of second order symmetric tensors on $R^{d}$. Let $\Omega \subset R^{d}$ be open, connected, and bounded with a Lipschitz boundary $\Gamma$ that is divided into three disjoint measurable parts $\Gamma_{1}, \Gamma_{2}$, and $\Gamma_{3}$ such that meas $\left(\Gamma_{1}\right)>0$. Let $L^{2}(\Omega)$ be the Lebesgue space of 2-integrable functions and $W^{k, p}(\Omega)$ the Sobolev space of functions whose weak derivatives of orders less than or equal to $k$ are $p$-integrable on $\Omega$. Let $H^{k}(\Omega)=W^{k, 2}(\Omega)$.

Since the boundary is Lipschitz continuous, the outward unit normal which is denoted by $v$ exists a.e. on $\Gamma$. For $T>0$, and let $\bar{I} \doteq[0, T]$ be the bounded time interval of interest. Let $\mathfrak{R}(u)$ be the range of displacement $u$. Since the body is clamped on $\Gamma_{1}$, the displacement field vanishes there. Surface traction of density $f_{2}$ acts on $\Gamma_{2}$ and a body force of density $f_{0}$ is applied in $\Omega$. The contact is bilateral, that is, the normal displacement $u_{v}$ vanishes on $\Gamma_{3}$ at any time. follows:

The canonical inner products and corresponding norms on $\mathcal{R}^{d}$ and $\mathcal{S}^{d}$ are defined as

$$
\begin{aligned}
& u \cdot v=u_{i} v_{i}, \quad\|v\|=(v \cdot v)^{1 / 2}, \quad \forall u, v \in R^{d}, \\
& \sigma \cdot \tau=\sigma_{i j} \tau_{i j}, \quad\|\tau\|=(\tau \cdot \tau)^{1 / 2}, \quad \forall \sigma, \tau \in \mathcal{S}^{d} .
\end{aligned}
$$

Everywhere in the sequel the index $i$ and $j$ run between 1 and $d$ and the summation convention over repeated indices is implied.

In the following we denote

$$
\begin{aligned}
& H=\left\{v=\left(v_{1}, v_{2}, \ldots, v_{d}\right)^{T} \mid v_{i} \in L^{2}(\Omega), 1 \leq i \leq d\right\}=L^{2}(\Omega)^{d},(u, v)_{H}=\int_{\Omega} u_{i}(x) v_{i}(x) d x, \\
& Q=\left\{\tau=\left(\tau_{i j}\right) \mid \tau_{i j}=\tau_{j i} \in L^{2}(\Omega), 1 \leq i, j \leq d\right\}=L^{2}(\Omega)_{s}^{d \times d},(\sigma, \tau)_{Q}=\int_{\Omega} \sigma_{i, j}(x) \tau_{i, j}(x) d x, \\
& Q_{1}=\Omega \times I,
\end{aligned}
$$




$$
\begin{aligned}
& H_{1}=\left\{v=\left(v_{1}, v_{2}, \ldots, v_{d}\right)^{T} \mid v_{i} \in H^{1}(\Omega), 1 \leq i \leq d\right\}=H^{1}(\Omega)^{d}, \\
& V=\left\{v \in H_{1} \mid v=0 \text { on } \Gamma_{1}\right\}, \\
& V_{1}=\left\{v \in V \mid v_{v}=0 \text { on } \Gamma_{3}\right\},
\end{aligned}
$$

where $H$ and $Q$ are Hilbert spaces with the canonical inner products. The associated norms on the spaces will be denoted by $\|\cdot\|_{H}$ and $\|\cdot\|_{Q}$, respectively.

Define

$$
(u, v)_{H_{1}}=(u, v)_{H}+(\varepsilon(u), \varepsilon(v))_{Q^{\prime}} \quad\|v\|_{H_{1}}=\sqrt{(v, v)_{H_{1}}}, \quad \forall u, v \in H_{1}
$$

It is easy to verify that $\left(H_{1},\|\cdot\|_{H_{1}}\right)$ is a real Hilbert space. Since $V$ is a closed subspace of the space $H_{1}$ and meas $\left(\Gamma_{1}\right)>0$, the following Korn's inequality holds:

$$
\|\varepsilon(v)\|_{Q} \geq \imath\|v\|_{H_{1}}, \quad \forall v \in V
$$

where $\iota$ denotes a positive constant depending only on $\Omega$ and $\Gamma_{1}$. We define the inner product $(\cdot, \cdot)_{V}$ and the norm $\|\cdot\|_{V}$ on $V$ by

$$
(u, v)_{V}=(\varepsilon(u), \varepsilon(v))_{Q^{\prime}} \quad\|v\|_{V}=\|\varepsilon(v)\|_{Q^{\prime}} \quad \forall u, v \in V
$$

It follows that $\|\cdot\|_{H_{1}}$ and $\|\cdot\|_{V}$ are equivalent norms on $V$. Thus, $\left(V,\|\cdot\|_{V}\right)$ is a real Hilbert space and $V_{1}$ is also a real Hilbert space under the inner product of the space $V$ given by (2.5).

For every element $v \in H_{1}$, we also use the notation $v$ for the trace of $v$ on $\Gamma$ and we denote by $v_{v}$ and $v_{\tau}$ the normal and the tangential components of $v$ on $\Gamma$ given by

$$
v_{v}=v \cdot v, \quad v_{\tau}=v-v_{v} v
$$

We also denote by $\sigma_{v}$ and $\sigma_{\tau}$ the normal and the tangential traces of a function $\sigma \in Q$, and we recall that when $\sigma$ is a regular function, that is, $\sigma \in C^{1}(\bar{\Omega})_{s}^{d \times d}$, then

$$
\sigma_{v}=(\sigma v) \cdot v, \quad \sigma_{\tau}=\sigma v-\sigma_{v} v,
$$

and the following Green's formula holds:

$$
(\sigma, \varepsilon(v))_{Q}+(\operatorname{Div} \sigma, v)_{H}=\int_{\Gamma} \sigma v \cdot v d a, \quad \forall v \in H_{1}
$$

We model the friction with Tresca's friction law, where the friction bound $g$ is assumed to depend on the accumulated slip of the surface. In this model we try to incorporate changes 
in the contact surface structure resulted from sliding. Therefore, $g=g\left(t, S_{t}(\dot{u})\right)$ on $\Gamma_{3} \times I$ with $S_{t}(\dot{u})(x)$ being the accumulated slip at the point $x$ on $\Gamma_{3}$ over the time period $\bar{I}$ as

$$
S_{t}(\dot{u})=\int_{0}^{t}\left\|\dot{u}_{\tau}(s)\right\| d s, \quad t \in \bar{I}
$$

It follows that $\left\|\sigma_{\tau}\right\| \leq g\left(S_{t}(\dot{u})\right)$ on $\Gamma_{3}$. When the strict inequality holds, the material point is in the stick zone: $\dot{u}_{\tau}=0$, while when the equality holds, $\left\|\sigma_{\tau}\right\|=g\left(S_{t}(\dot{u})\right)$, the material point is in the slip zone: $\sigma_{\tau}=-\lambda \dot{u}_{\tau}$ for some $\lambda>0$.

Let $r$ be a constant satisfying $0<r<T$ and set $Q_{-r}=\Omega \times(-r, 0)$. Let $\mathfrak{B}$ be the Borel $\sigma$ algebra of the interval $[-r, 0]$ and $\mu(\cdot)$ be a given finite signed measure defined on $([-r, 0], \mathfrak{B})$. Zhu [22] defined the time-delay operator $G$ as follows: for any $h \in L^{2}(\Omega \times(-r, \infty))_{S}^{d \times d}$,

$$
(G h)(t, x) \doteq \int_{-r}^{0} h(t+\theta, x) \mu(d \theta) .
$$

In order to make the above integral coherent, we always take the integrand to be a Borel correction of $h$ (by which we mean a Borel measurable function that is equal to $h$ almost everywhere).

Some special cases of the operator $G$ are as follows:

(i) Let $\Omega_{1}=\left\{\omega_{1}, \omega_{2}, \ldots, \omega_{n}, \ldots\right\}, \mathcal{L}_{1}=2^{\Omega_{1}}$, and

$$
\mu_{1}(A)=\sum_{\omega_{i} \in A} \mu_{1}\left(\omega_{i}\right)=\sum_{\omega_{i} \in A} p_{i}, \quad A \in \mathcal{L}_{1}
$$

where $\mu_{1}\left(\omega_{i}\right)=p_{i}, i=1,2, \ldots, n, \ldots, p_{i} \in \mathcal{R}^{+}$and $\mu_{1}(\emptyset)=0$. Then it is easy to see that

$$
(G h)(t, x)=\int_{-r}^{0} h(t+\theta, x) \mu_{1}(d \theta)=\sum_{\omega_{i} \in(-r, 0)} p_{i} h\left(t+\omega_{i}, x\right),
$$

which can be used to describe the countably many discrete delays.

(ii) Let $\Omega_{2}=\mathcal{R}, \mathcal{L}_{2}$ be a $\sigma$-algebra of $\Omega_{2}, m$ a Lebesgue measure, $f$ a Lebesgue measurable function, and

$$
\mu_{2}(A)=\int_{A} f(x) d m, \quad A \in \mathcal{L}_{2}
$$

Then

$$
(G h)(t, x)=\int_{-r}^{0} h(t+\theta, x) \mu_{2}(d \theta)=\int_{-r}^{0} h(t+\theta, x) \int_{d \theta} f(x) d m .
$$


Moreover, letting $f \equiv 1$, then

$$
(G h)(t, x)=\int_{-r}^{0} h(t+\theta, x) \mu_{2}(d \theta)=\int_{-r}^{0} h(t+\theta, x) d \theta .
$$

Remark 2.1. It is easy to see that, for any $h \in L^{2}(\Omega \times(-r, \infty))_{S}^{d \times d}, G h$ as an element in $L^{2}(\Omega \times$ $(0, \infty))_{S}^{d \times d}$ is independent of the choices of Borel corrections for $h$.

Remark 2.2. Since $\mu$ is a very general regular measure, (2.10) can be used in many cases such as finitely many and countably many discrete delays. At this stage, we note that (2.10) contains a very wide class of time-delay operators.

The following lemma is a fundamental result for operator $G$.

Lemma 2.3 (see [22]). For $h \in L^{2}\left((-r, \infty) \times \Omega ; \mathcal{R}^{d}\right)$, we have $G h \in L^{2}\left((0, \infty) \times \Omega ; \mathcal{R}^{d}\right)$. Furthermore, for any $g \in L^{2}\left((0, \infty) \times \Omega ; R^{d}\right), 0 \leq s \leq+\infty$ and $0 \leq s_{0} \leq r$, the following inequality holds:

$$
\begin{aligned}
\left|\int_{\Omega} d x \int_{0}^{s+s_{0}}(G h) \cdot g d t\right| \leq & \frac{1}{2} \int_{\Omega} d x \int_{0}^{s+s_{0}}\|g\|^{2} d t+\frac{1}{2}|\mu|([-r, 0])^{2} \int_{\Omega} d x \int_{-r}^{s}\|h\|^{2} d t \\
& +\frac{1}{2}|\mu|([-r, 0])|\mu|\left(\left[-s_{0}, 0\right]\right) \int_{\Omega} d x \int_{-r}^{s+s_{0}}\|h\|^{2} d t .
\end{aligned}
$$

Now we consider the contact problem. For any $\tilde{u} \in \mathfrak{R}(u)$, based on (2.10), we derive the time-delay operator $\mathcal{G}$ of the form

$$
(\mathcal{G} h)(t, \varepsilon(\widetilde{u})) \doteq \int_{-r}^{0} h(t+\theta, \varepsilon(\widetilde{u})) \mu(d \theta) .
$$

Remark 2.4. Replacing $x$ with $\varepsilon(\tilde{u})$ in (2.10) and letting $s_{0}=0$ and $g=G h$ in Lemma 2.3, it is easy to know that

$$
\|\mathcal{G}\| \leq|\mu|([-r, 0])
$$

Under the previous assumptions, the classical formulation of the frictional contact problem with total slip dependent friction bound and the time-delay is as follows. For any $\widetilde{u} \in \mathfrak{R}(u)$, find a displacement field $u: \Omega \times \bar{I} \rightarrow \mathcal{R}^{d}$ and a stress field $\sigma: \Omega \times \bar{I} \rightarrow \mathcal{S}^{d}$ such that

$$
\begin{gathered}
\sigma(t)=\mathcal{A}(t, \varepsilon(\dot{u}(t)))+\mathcal{B}(t, \varepsilon(u(t)))+\mathcal{G} h(t, \varepsilon(\tilde{u})) \text { in } \Omega \times \bar{I}, \\
\operatorname{Div} \sigma(t)+f_{0}(t)=0 \quad \text { in } \Omega \times \bar{I}, \\
u(t)=0 \quad \text { on } \Gamma_{1} \times \bar{I},
\end{gathered}
$$


Mathematical Problems in Engineering

$$
\begin{gathered}
\sigma(t) v=f_{2}(t) \quad \text { on } \Gamma_{2} \times \bar{I}, \\
u_{v}(t)=0, \quad\left\|\sigma_{\tau}(t)\right\| \leq g\left(t, S_{t}(\dot{u}(t))\right) \quad \text { on } \Gamma_{3} \times \bar{I}, \\
u(0)=u_{0} \quad \text { in } \Omega .
\end{gathered}
$$

We present a short description of the equations and conditions in Problems (2.19)(2.24). For more details and mechanical interpretation, we refer to $[1,16]$. Here (2.19) represents the viscoelastic constitutive law in which $\mathcal{A}, \mathbb{B}$, and $\mathcal{G}$ are given nonlinear operators, called the viscosity operator, elasticity operator, and time-delay operator, respectively. The prime represents the derivative with respect to the time variable, and therefore $\dot{u}$ represents the velocity field. Note that the explicit dependence of the viscosity, elasticity, and time-delay operators $\mathcal{A}, \mathbb{B}$, and $\mathcal{G}$ with respect to the time variable means that the model involve the situations when the properties of the material depend on the temperature, that is, its evolution in time is prescribed. Equality (2.20) represents the equilibrium equation where $\operatorname{Div} \sigma=\left(\sigma_{i j, j}\right)$ represents the divergence of stress. Conditions (2.21) and (2.22) are the displacement and traction boundary conditions, respectively. Equation (2.23) represents the frictional contact conditions and (2.24) is the initial condition in which the function $u_{0}$ denotes the initial displacement field.

In the study of mechanical problems (2.19)-(2.24), we assume that $\mathcal{A}, \mathbb{B}, g$, and $h$ satisfy the following conditions.

$\mathrm{H}(\mathcal{A}): \mathcal{A}: Q_{1} \times \mathcal{S}^{d} \rightarrow \mathcal{S}^{d}$ is an operator such that

(i) $\left\|\mathscr{A}\left(x, t_{1}, \varepsilon_{1}\right)-\mathcal{A}\left(x, t_{2}, \varepsilon_{2}\right)\right\|_{Q} \leq L\left(\left|t_{1}-t_{2}\right|+\left\|\varepsilon_{1}-\varepsilon_{2}\right\|_{Q}\right)$, for all $\varepsilon_{1}, \varepsilon_{2} \in \mathcal{S}^{d}, t_{1}, t_{2} \in \bar{I}$, a.e. $x \in \Omega$ with $L>0$;

(ii) $\left(\left(\mathcal{A}\left(x, t, \varepsilon_{1}\right)-\mathcal{A}\left(x, t, \varepsilon_{2}\right)\right),\left(\varepsilon_{1}-\varepsilon_{2}\right)\right)_{Q} \geq M\left\|\varepsilon_{1}-\varepsilon_{2}\right\|_{Q^{\prime}}^{2}$, for all $\varepsilon_{1}, \varepsilon_{2} \in \mathcal{S}^{d}$, a.e. $(x, t) \in Q_{1}$ with $M>0$;

(iii) for any $\varepsilon \in \mathcal{S}^{d},(x, t) \mapsto \mathcal{A}(x, t, \varepsilon)$ is measurable on $Q_{1}$;

(iv) the mapping $(x, t) \mapsto \mathcal{A}(x, t, 0) \in L^{2}\left(Q_{1}\right)^{d \times d}$.

$\mathrm{H}(\boldsymbol{B}): \mathbb{B}: Q_{1} \times \mathcal{S}^{d} \rightarrow \mathcal{S}^{d}$ is an operator such that

(i) $\left\|\mathbb{B}\left(x, t, \varepsilon_{1}\right)-\mathcal{B}\left(x, t, \varepsilon_{2}\right)\right\|_{Q} \leq L_{1}\left\|\varepsilon_{1}-\varepsilon_{2}\right\|_{Q}$, for all $\varepsilon_{1}, \varepsilon_{2} \in \mathcal{S}^{d}$, a.e. $(x, t) \in Q_{1}$ with $L_{1}>0$;

(ii) for any $\varepsilon \in \mathcal{S}^{d}, \quad(x, t) \mapsto B(x, t, \varepsilon)$ is measurable on $Q$;

(iii) the mapping $(x, t) \mapsto \mathcal{B}(x, t, 0) \in L^{2}\left(Q_{1}\right)^{d \times d}$.

$\mathrm{H}(g): g: \Gamma_{3} \times I \times \mathcal{R} \rightarrow \mathcal{R}^{+}$is an operator such that

(i) there exists $L_{2}>0$ such that for all $t_{1}, t_{2} \in \bar{I}, u_{1}, u_{2} \in \mathcal{R}, x \in \Omega, \mid g\left(x, t_{1}, u_{1}\right)-$ $g\left(x, t_{2}, u_{2}\right) \mid \leq L_{2}\left(\left|t_{1}-t_{2}\right|+\left|u_{1}-u_{2}\right|\right)$;

(ii) for any $u \in \mathcal{R},(x, t) \mapsto g(x, t, u)$ is measurable;

(iii) the mapping $(x, t) \mapsto g(x, t, 0) \in L^{2}\left(\Gamma_{3} \times I\right)$;

(iv) $\left\|\sigma_{\tau}\right\|<g\left(t, S_{t}(\dot{u}(t))\right) \Rightarrow \dot{u}_{\tau}=0,\left\|\sigma_{\tau}\right\|=g\left(t, S_{t}(\dot{u}(t))\right) \Rightarrow$ exists $\lambda \geq 0$ such that $\sigma_{\tau}=$ $-\lambda \dot{u}_{\tau}$.

$\mathrm{H}(h): h: Q_{1} \times \mathcal{S}^{d} \rightarrow \mathcal{S}^{d}$ is an operator such that

(i) $\left\|h\left(x, t, \varepsilon_{1}\right)-h\left(x, t, \varepsilon_{2}\right)\right\|_{Q} \leq L_{3}\left\|\varepsilon_{1}-\varepsilon_{2}\right\|_{Q}$, for all $\varepsilon_{1}, \varepsilon_{2} \in \mathcal{S}^{d}$, a.e. $(x, t) \in Q_{1}$ with $L_{3}>0$; 
(ii) for any $\varepsilon \in \mathcal{S}^{d},(x, t) \mapsto h(x, t, \varepsilon)$ is measurable on $Q_{1}$;

(iii) $\left(h\left(x, t, \varepsilon_{1}\right)-h\left(x, t, \varepsilon_{2}\right),\left(\varepsilon_{1}-\varepsilon_{2}\right)\right)_{Q} \geq M^{\prime}\left\|\varepsilon_{1}-\varepsilon_{2}\right\|_{Q^{\prime}}^{2}$ for all $\varepsilon_{1}, \varepsilon_{2} \in \mathcal{S}^{d}$, a.e. $(x, t) \in Q_{1}$ with $M^{\prime}>0$;

(iv) the mapping $(x, t) \mapsto h(x, t, 0) \in L^{2}\left(Q_{1}\right)^{d \times d}$.

In the following, we provide an elementary example of the mechanical problem which hold the constitutive law equation (2.19).

Example 2.5. Let $\mathcal{A}$ and $B$ be nonlinear operators which describe the viscous and the elastic properties of the material and satisfy the conditions $\mathrm{H}(\mathcal{A})$ and $\mathrm{H}(\mathbb{B})$, respectively, while $\mathcal{C}$ is the linear relaxation operator. The following example is assumed to be the Kelvin-Voigt viscoelastic constitutive law with long memory of the form

$$
\sigma(t)=\mathscr{A}(t, \varepsilon(\dot{u}(t)))+\mathcal{B}(t, \varepsilon(u(t)))+\int_{0}^{t} \mathcal{C}(t-s) \varepsilon(u(s)) d s
$$

which represents a constitutive equation of the form (2.19).

Contact problems involving viscoelastic materials with long memory have been studied in $[25,26]$. For more detail on the long memory models, we refer to [27, 28].

The famous time-temperature superposition principle tells us that when materials are applied with the alternating stress, the reaction time is an inverse proportion to the effect of the frequency. Hence, the influence of increasing the time (or reducing the frequency) and elevating temperature to materials is equivalent.

The sinusoidally driven indentation test was shown to be effective for viability characterization of articular cartilage. Based on the viscoelastic correspondence principle, Argatov [5] described the mechanical response of the articular cartilage layer in the framework of viscoelastic model. Using the asymptotic modeling approach, Argatov analyzed and interpreted the results of the indentation test. Now, deriving from the (30) and (115) in [5], and noting the relationship between time and frequency, we write the viscoelastic constitutive law in the following form:

$$
\sigma(t)=\frac{a_{1} t^{2}}{a_{2}+t^{2}} \varepsilon(\dot{u}(t))+\frac{b_{1}+b_{2} t^{2}}{b_{3}+t^{2}} \varepsilon(u(t))
$$

where $a_{1}, a_{2}$ and $b_{1}, b_{2}, b_{3}$ are some parameters which rely on the characteristic relaxation time of strain under an applied step in stress, the equilibrium elastic modulus, and the glass elastic modulus.

It is easy to verify that $\mathcal{A}(t, \varepsilon(\dot{u}(t))) \doteq\left(a_{1} t^{2} /\left(a_{2}+t^{2}\right)\right) \varepsilon(\dot{u}(t))$ and $B(t, \varepsilon(u(t))) \doteq\left(\left(b_{1}+\right.\right.$ $\left.\left.b_{2} t^{2}\right) /\left(b_{3}+t^{2}\right)\right) \varepsilon(u(t))$ satisfy the assumption $\mathrm{H}(\mathcal{A})$ and $\mathrm{H}(\mathbb{B})$, respectively.

Next, we denote by $f(t)$ the element of $V_{1}$ given by

$$
(f(t), v)_{V}=\left(f_{0}(t), v\right)_{H}+\left(f_{2}(t), v\right)_{L^{2}\left(\Gamma_{2}\right)^{d}}, \quad \forall v \in V_{1} \text {, a.e. } t \in \bar{I}
$$


When we assume that the body force and surface traction satisfy $f_{0} \in C(\bar{I} ; H)$ and $f_{2} \in C\left(\bar{I} ; L^{2}\left(\Gamma_{2}\right)\right)$, we can get

$$
f \in C\left(\bar{I} ; V_{1}\right)
$$

Let $j: I \times L^{2}\left(\Gamma_{3}\right) \times V_{1} \rightarrow \mathcal{R}$ be the functional defined as follows:

$$
j(t, v ; w)=\int_{\Gamma_{3}} g(t, v)\left\|w_{\tau}\right\| d a, \quad \forall v \in L^{2}\left(\Gamma_{3}\right), w \in V_{1} .
$$

We notice that, by the assumption $\mathrm{H}(g)$, the integral in (2.29) is well defined.

Lemma 2.6 (Gronwall's inequality). Assume that $f, g \in C[a, b]$ satisfy

$$
f(t) \leq g(t)+c \int_{a}^{t} f(s) d s, \quad t \in[a, b]
$$

where $c>0$ is a constant. Then

$$
f(t) \leq g(t)+c \int_{a}^{t} g(s) e^{c(t-s)} d s, \quad t \in[a, b]
$$

Moreover, if $g$ is nondecreasing, then

$$
f(t) \leq g(t) e^{c(t-a)}, \quad t \in[a, b]
$$

Proceeding in a standard way with these notations, we combine (2.8)-(2.24) to obtain the following variational formulation.

Problem 1. Find a displacement $u: \bar{I} \rightarrow V_{1}$ such that (2.24) holds and

$$
\begin{aligned}
& (\mathcal{A}(t, \varepsilon(\dot{u}(t))), \varepsilon(v-\dot{u}(t)))_{Q}+(\mathbb{B}(t, \varepsilon(u(t))), \varepsilon(v-\dot{u}(t)))_{Q}+(\mathcal{G} h(t, \varepsilon(\tilde{u})), \varepsilon(v-\dot{u}(t)))_{Q} \\
& \quad+j\left(t, S_{t}(\dot{u}) ; v\right)-j\left(t, S_{t}(\dot{u}) ; \dot{u}(t)\right) \geq(f(t), v-\dot{u}(t))_{V}, \quad \forall v \in V_{1}, \tilde{u} \in \Re(u) \text {, a.e. } t \in \bar{I} .
\end{aligned}
$$

We first introduce the following problem.

Problem 2. Find a displacement $u: \bar{I} \rightarrow V_{1}$ such that (2.24) holds and

$$
\begin{aligned}
& (\mathcal{A}(t, \varepsilon(\dot{u}(t))), \varepsilon(v-\dot{u}(t)))_{Q}+(\mathcal{G} h(t, \varepsilon(\tilde{u})), \varepsilon(v-\dot{u}(t)))_{Q}+j\left(t, S_{t}(\dot{u}) ; v\right)-j\left(t, S_{t}(\dot{u}) ; \dot{u}(t)\right) \\
& \geq(f(t), v-\dot{u}(t))_{V}, \quad \forall v \in V_{1}, \tilde{u} \in \mathfrak{R}(u), \text { a.e. } t \in \bar{I} .
\end{aligned}
$$


For solving the above problems, we derive some results for an elliptic variational inequality of the second kind: Given $f \in X$, find $u \in V$ such that

$$
(A(t, u), v-u)_{V}+j(v)+j(u) \geq(f, v-u)_{V}, \quad \forall v \in V, \text { a.e. } t \in \bar{I} .
$$

Lemma 2.7 (see [1]). Let $j: V \rightarrow \bar{R}$ be a proper, convex, and lower semicontinuous functional. Then for any $f \in V$, there exists a unique element $u:=\operatorname{Prox}_{j}(f)$ such that

$$
u \in V, \quad(u, v-u)_{V}+j(v)-j(u) \geq(f, v-u)_{V^{\prime}} \quad \forall v \in V \text {, a.e. } t \in \bar{I} .
$$

Lemma 2.8. Let $V$ be a Hilbert space. Assume that $H(\mathcal{A})$ holds and $j: V \rightarrow \overline{\mathcal{R}}$ is a proper, convex, lower semicontinuous functional. Then for any $f \in V$, variational inequality (2.35) has a unique solution.

Proof. For any $f \in V$, let $\rho>0$ be a parameter to be chosen later. Since $\rho j: V \rightarrow \overline{\mathcal{R}}$ is again a proper, convex, and lower semicontinuous functional, we can define an operator $T: \bar{I} \times V \rightarrow$ $V$ by

$$
T(t, v)=\operatorname{Prox}_{\rho j}(\rho f-\rho A(t, v)+v), \quad \forall v \in V, \text { a.e. } t \in \bar{I},
$$

where $(A(t, u), v)_{V} \doteq(\mathcal{A}(x, t, \varepsilon(u)), \varepsilon(v))_{Q}$ (see (2.5)). We will show that with a suitable choice of $\rho$ the operator $T$ is a contractive mapping on $\bar{I} \times V$. To this end, let $u, v \in V$. Since Prox is a nonexpansive mapping, it follows from (2.37) that

$$
\begin{aligned}
\|T(t, u)-T(t, v)\|_{V}^{2} & \leq\|u-v-\rho(A(t, u)-A(t, v))\|_{V}^{2} \\
& =\|u-v\|_{V}^{2}-2 \rho(A(t, u)-A(t, v), u-v)_{V}+\rho^{2}\|A(t, u)-A(t, v)\|_{V}^{2} .
\end{aligned}
$$

Using the assumption $\mathrm{H}(\mathscr{A})$ and $(2.5)$, we obtain

$$
\|T(t, u)-T(t, v)\|_{V}^{2} \leq\left(1-2 \rho M+\rho^{2} L^{2}\right)\|u-v\|_{V}^{2} .
$$

If $0 \leq \rho \leq 2 M / L^{2}$, then

$$
0<1-2 \rho M+\rho^{2} L^{2}<1
$$

Taking

$$
\alpha=\left(1-2 \rho M+\rho^{2} L^{2}\right)^{1 / 2}
$$

we deduce that $\alpha \in(0,1)$ and

$$
\|T(t, u)-T(t, v)\|_{V}^{2} \leq \alpha\|u-v\|_{V}^{2},
$$


which shows that $T: \bar{I} \times V \rightarrow V$ is a contractive mapping. Therefore, $T$ has a fixed point $u$, that is,

$$
u=\operatorname{Prox}_{\rho j}(\rho f-\rho A(t, u)+u), \quad \text { a.e. } t \in \bar{I},
$$

which implies

$$
(u, v-u)_{V}+\rho j(v)-\rho j(u) \geq(\rho f-\rho A(t, u)+u, v-u)_{V}, \quad \forall v \in V \text {, a.e. } t \in \bar{I} .
$$

It follows that

$$
\rho\left[(A(t, u), v-u)_{V}+j(v)-j(u)\right] \geq \rho(f, v-u)_{V}, \quad \forall v \in V, \text { a.e. } t \in \bar{I} .
$$

Since $\rho>0$, we deduce from the above inequality that $u$ is a solution of variational inequality (2.35).

To show the uniqueness, we assume that there exist two solutions $u_{1}, u_{2} \in V$ of variational inequality (2.35). Then for any $v \in V$ and a.e. $t \in \bar{I}$, we have

$$
\begin{aligned}
& \left(A\left(t, u_{1}\right), v-u_{1}\right)_{V}+j(v)+j\left(u_{1}\right) \geq\left(f, v-u_{1}\right)_{V} \\
& \left(A\left(t, u_{2}\right), v-u_{2}\right)_{V}+j(v)+j\left(u_{2}\right) \geq\left(f, v-u_{2}\right)_{V} .
\end{aligned}
$$

Since $j$ is proper, we know that $j\left(u_{1}\right)<\infty$ and $j\left(u_{2}\right)<\infty$. Taking $v=u_{2}$ in the first inequality and $v=u_{1}$ in the second one and adding the corresponding inequalities, we get

$$
\left(A\left(t, u_{1}\right)-A\left(t, u_{2}\right), u_{1}-u_{2}\right)_{V} \leq 0 .
$$

Using (2.5) and $\mathrm{H}(\mathcal{A})$, we obtain that $u_{1}=u_{2}$, which completes the proof of Lemma 2.8.

Remark 2.9. Lemma 2.8 is a generalization of Theorem 4.1 of [1].

\section{Main Results}

In this section, we present an existence and uniqueness result concerned with the solution of Problem 1. Throughout this section, we assume that $\mathrm{H}(\mathcal{A}), \mathrm{H}(\mathbb{B}), \mathrm{H}(g), \mathrm{H}(h)$, and (2.28) hold.

Theorem 3.1. Problem 2 has a unique solution $u \in C^{1}\left(\bar{I} ; V_{1}\right)$.

The proof of Theorem 3.1 is based on fixed point arguments and is established in several steps. Let $\eta \in C(\bar{I} ; Q)$ and $\xi \in C\left(\bar{I} ; V_{1}\right)$ be arbitrarily given. We consider the following auxiliary variational problem.

Problem 3. Find $w_{\eta \xi}: \bar{I} \rightarrow V_{1}$ such that for any $v \in V_{1}$

$$
\begin{aligned}
& \left(\mathcal{A}\left(t, \varepsilon\left(w_{\eta \xi}(t)\right)\right), \varepsilon\left(v-w_{\eta \xi}(t)\right)\right)_{Q}+\left(\eta(t), \varepsilon\left(v-w_{\eta \xi}(t)\right)\right)_{Q}+j\left(t, S_{t}(\xi) ; v\right)-j\left(t, S_{t}(\xi) ; w_{\eta \xi}(t)\right) \\
& \quad \geq\left(f(t), v-w_{\eta \xi}(t)\right)_{V^{\prime}}, \quad \text { a.e. } t \in \bar{I}
\end{aligned}
$$


Lemma 3.2. There exists a unique solution $w_{\eta \xi} \in C\left(\bar{I} ; V_{1}\right)$ to Problem 3.

Proof. For each fixed $t \in \bar{I}$, in terms of hypotheses (2.9), $\mathrm{H}(\mathcal{A}), \mathrm{H}(g)$, and (2.29), Problem 3 is an elliptic variational inequality on $Q$. It follows from Lemma 2.8 that Problem 3 is uniquely solvable. Let $w_{\eta \xi}(t) \in V_{1}$ be the unique solution of Problem 3. Now we show that $w_{\eta \xi}(t) \in$ $C\left(\bar{I}, V_{1}\right)$.

Suppose that $t_{1}, t_{2} \in \bar{I}$. For simplicity we write $w_{\eta \xi}\left(t_{i}\right)=w_{i}, \eta\left(t_{i}\right)=\eta_{i}$ and $f\left(t_{i}\right)=f_{i}$ with $i=1$, 2. Using (3.1) for $t=t_{1}, t_{2}$, we have

$$
\begin{aligned}
& \left(\mathcal{A}\left(t_{1}, \varepsilon\left(w_{1}\right)\right), \varepsilon\left(v-w_{1}\right)\right)_{Q}+\left(\eta_{1}, \varepsilon\left(v-w_{1}\right)\right)_{Q}+j\left(t_{1}, S_{t_{1}}(\xi) ; v\right)-j\left(t_{1}, S_{t_{1}}(\xi) ; w_{1}\right) \\
& \quad \geq\left(f_{1}, v-w_{1}\right)_{V^{\prime}} \\
& \left(\mathcal{A}\left(t_{2}, \varepsilon\left(w_{2}\right)\right), \varepsilon\left(v-w_{2}\right)\right)_{Q}+\left(\eta_{2}, \varepsilon\left(v-w_{2}\right)\right)_{Q}+j\left(t_{2}, S_{t_{2}}(\xi) ; v\right)-j\left(t_{2}, S_{t_{2}}(\xi) ; w_{2}\right) \\
& \quad \geq\left(f_{2}, v-w_{2}\right)_{V} .
\end{aligned}
$$

By adding two inequalities with $v=w_{2}$ in (3.2) and $v=w_{1}$ in (3.3), we get

$$
\begin{aligned}
& \left(\mathcal{A}\left(t_{1}, \varepsilon\left(w_{1}\right)\right)-\mathcal{A}\left(t_{2}, \varepsilon\left(w_{2}\right)\right), \varepsilon\left(w_{1}-w_{2}\right)\right)_{Q} \\
& \quad \leq\left(f_{1}-f_{2}, w_{1}-w_{2}\right)_{V}+\left(\eta_{1}-\eta_{2}, \varepsilon\left(w_{1}-w_{2}\right)\right)_{Q}+D\left(t_{1}, t_{2}, \xi, w_{1}, w_{2}\right),
\end{aligned}
$$

where

$$
D\left(t_{1}, t_{2}, \xi, w_{1}, w_{2}\right)=j\left(t_{1}, S_{t_{1}}(\xi) ; w_{2}\right)-j\left(t_{1}, S_{t_{1}}(\xi) ; w_{1}\right)+j\left(t_{2}, S_{t_{2}}(\xi) ; w_{1}\right)-j\left(t_{2}, S_{t_{2}}(\xi) ; w_{2}\right) .
$$

It implies that

$$
\begin{aligned}
& \left(\mathcal{A}\left(t_{1}, \varepsilon\left(w_{1}\right)\right)-\mathcal{A}\left(t_{1}, \varepsilon\left(w_{2}\right)\right), \varepsilon\left(w_{1}-w_{2}\right)\right)_{Q} \\
& \leq\left(\mathcal{A}\left(t_{1}, \varepsilon\left(w_{2}\right)\right)-\mathcal{A}\left(t_{2}, \varepsilon\left(w_{2}\right)\right), \varepsilon\left(w_{1}-w_{2}\right)\right)_{Q} \\
& \quad+D\left(t_{1}, t_{2}, \xi, w_{1}, w_{2}\right)+\left(\eta_{1}-\eta_{2}, \varepsilon\left(w_{1}-w_{2}\right)\right)_{Q}+\left(f_{1}-f_{2}, w_{1}-w_{2}\right)_{V} .
\end{aligned}
$$

By $\mathrm{H}(\mathcal{A})$, we get

$$
\begin{gathered}
\left(\mathcal{A}\left(t_{1}, \varepsilon\left(w_{1}\right)\right)-\mathcal{A}\left(t_{1}, \varepsilon\left(w_{2}\right)\right), \varepsilon\left(w_{1}-w_{2}\right)\right)_{Q} \geq M\left\|w_{1}-w_{2}\right\|_{V}^{2} \\
\left\|\mathcal{A}\left(t_{1}, \varepsilon\left(w_{2}\right)\right)-\mathcal{A}\left(t_{2}, \varepsilon\left(w_{2}\right)\right)\right\|_{Q} \leq L\left|t_{1}-t_{2}\right| .
\end{gathered}
$$

Constituting a trace operator $\gamma: V \rightarrow L^{2}\left(\Gamma_{3}\right)$ that $\gamma v=\left.v\right|_{\Gamma_{3}}$, since $\gamma$ is a linear continuous operator, it implies that there exists a constant $c>0$ such that

$$
\|v\|_{L^{2}\left(\Gamma_{3}\right)} \leq c\|v\|_{V}
$$


It follows from (2.9), (2.29), (3.8), and $\mathrm{H}(g)$ that

$$
D\left(t_{1}, t_{2}, \xi, w_{1}, w_{2}\right) \leq L_{2}\left(1+\|\xi\|_{C(\bar{I} ; V)}\right)\left|t_{1}-t_{2}\right| \cdot\left\|w_{1}-w_{2}\right\|
$$

By (3.6)-(3.7) and (3.9), we have

$$
\left\|w_{1}-w_{2}\right\|_{V} \leq \frac{1}{M}\left(\left\|f_{1}-f_{2}\right\|_{V}+\left\|\eta_{1}-\eta_{2}\right\|_{Q}+\left|t_{1}-t_{2}\right|\left(L+L_{2}\left(1+\|\xi\|_{C(\bar{I} ; V)}\right)\right)\right),
$$

which implies that $w_{\eta \xi} \in C\left(\bar{I} ; V_{1}\right)$. This completes the proof of Lemma 3.2.

In order to get the unique solution of Problem 2, we derive the following operator $\Lambda_{\eta}: C\left(\bar{I} ; V_{1}\right) \rightarrow C\left(\bar{I} ; V_{1}\right)$ defined by

$$
\Lambda_{\eta} \xi=w_{\eta \xi}, \quad \forall \xi \in C\left(\bar{I} ; V_{1}\right)
$$

Lemma 3.3. For any $\eta \in C(\bar{I} ; Q)$, the operator $\Lambda_{\eta}$ has a unique fixed point $\xi_{\eta} \in C\left(\bar{I} ; V_{1}\right)$.

Proof. Let $\eta \in C(\bar{I} ; Q)$ and $\xi_{1}, \xi_{2} \in C\left(\bar{I} ; V_{1}\right)$. We denote by $w_{i}$ the solution of Problem 3 with $\xi=\xi_{i}$ for $i=1,2$. By an argument similar to that used in obtaining (3.6), we get

$$
\left(\mathcal{A}\left(t, \varepsilon\left(w_{1}\right)\right)-\mathcal{A}\left(t, \varepsilon\left(w_{2}\right)\right), \varepsilon\left(w_{1}-w_{2}\right)\right)_{Q} \leq D\left(t, \xi_{1}, \xi_{2}, w_{1}, w_{2}\right),
$$

where

$$
D\left(t, \xi_{1}, \xi_{2}, w_{1}, w_{2}\right)=j\left(t, S_{t}\left(\xi_{1}\right) ; w_{2}\right)-j\left(t, S_{t}\left(\xi_{1}\right) ; w_{1}\right)+j\left(t, S_{t}\left(\xi_{2}\right) ; w_{1}\right)-j\left(t, S_{t}\left(\xi_{2}\right) ; w_{2}\right) .
$$

Using (2.29), (3.8), (2.9), and $\mathrm{H}(g)$, we deduce that, for any $t \in \bar{I}$,

$$
D\left(t, \xi_{1}, \xi_{2}, w_{1}, w_{2}\right) \leq c_{1} \int_{0}^{t}\left\|\xi_{1}(s)-\xi_{2}(s)\right\|_{V} d s\left\|w_{1}(t)-w_{2}(t)\right\|_{V}
$$

where $c_{1}=c L_{2}$. By using the similar method in obtaining (3.10), we have

$$
\left\|w_{1}(t)-w_{2}(t)\right\|_{V} \leq \frac{c_{1}}{M} \int_{0}^{t}\left\|\xi_{1}(s)-\xi_{2}(s)\right\|_{V} d s, \quad \text { a.e. } t \in \bar{I}
$$

Since $w_{i}=w_{\eta \xi_{i}}=\Lambda_{\eta} \xi_{i}$, we rewrite the above inequality as

$$
\left\|\Lambda_{\eta} \xi_{1}(t)-\Lambda_{\eta} \xi_{2}(t)\right\|_{V} \leq \frac{c_{1}}{M} \int_{0}^{t}\left\|\xi_{1}(s)-\xi_{2}(s)\right\|_{V} d s, \quad \text { a.e. } t \in \bar{I}
$$


For $w \in C\left(\bar{I} ; V_{1}\right)$, let

$$
\|w\|_{\beta}=\max _{t \in I} e^{-\beta t}\|w(t)\|_{V}
$$

where $\beta>0$ is a constant which will be chosen later. Clearly, $\|\cdot\|_{\beta}$ defines a norm on the space $C\left(\bar{I} ; V_{1}\right)$ and

$$
\begin{aligned}
e^{-\beta t}\left\|\Lambda_{\eta} \xi_{1}(t)-\Lambda_{\eta} \xi_{2}(t)\right\|_{V} & \leq \frac{c_{1} e^{-\beta t}}{M} \int_{0}^{t} e^{\beta s} e^{-\beta s}\left\|\xi_{1}(s)-\xi_{2}(s)\right\|_{V} d s \\
& \leq \frac{c_{1} e^{-\beta t}}{M} \int_{0}^{t} e^{\beta s}\left\|\xi_{1}-\xi_{2}\right\|_{\beta} d s \leq \frac{c_{1}}{M \beta}\left\|\xi_{1}-\xi_{2}\right\|_{\beta}, \quad \text { a.e. } t \in \bar{I} .
\end{aligned}
$$

Thus,

$$
\left\|\Lambda_{\eta} \xi_{1}-\Lambda_{\eta} \xi_{2}\right\|_{\beta} \leq \frac{c_{1}}{M \beta}\left\|\xi_{1}-\xi_{2}\right\|_{\beta}
$$

and so the operator $\Lambda_{\eta}$ is a contraction on the space $C\left(\bar{I} ; V_{1}\right)$ endowed with the equivalent norm $\|\cdot\|_{\beta}$ if we choose $\beta$ such that $M \beta>c_{1}$. Therefore, the operator $\Lambda_{\eta}$ has a unique fixed point $\xi_{\eta} \in C\left(\bar{I} ; V_{1}\right)$, which completes the proof of Lemma 3.3.

In what follows, for any $\eta \in C(\bar{I} ; Q)$, we write

$$
w_{\eta}=w_{\eta \xi}
$$

By $\Lambda_{\eta} \xi_{\eta}=\xi_{\eta},(3.11)$, and (3.20), we have

$$
w_{\eta}=\xi_{\eta}
$$

Taking $\xi=\xi_{\eta}$ in (3.1) and using (3.20) and (3.21), we deduce that, for any $v \in V_{1}$,

$$
\begin{aligned}
& \left(\mathcal{A}\left(\mathrm{t}, \varepsilon\left(w_{\eta}(t)\right)\right), \varepsilon\left(v-w_{\eta}(t)\right)\right)_{Q}+\left(\eta, \varepsilon\left(v-w_{\eta}(t)\right)\right)_{Q} \\
& \quad+j\left(t, S_{t}\left(w_{\eta}\right) ; v\right)-j\left(t, S_{t}\left(w_{\eta}\right) ; w_{\eta}(t)\right) \geq\left(f(t), v-w_{\eta}(t)\right)_{V}, \quad \text { a.e. } t \in \bar{I} .
\end{aligned}
$$

Let $w_{\eta}: \bar{I} \rightarrow V_{1}$ be the function given by

$$
u_{\eta}(t)=\int_{0}^{t} w_{\eta}(x) d s+u_{0}, \quad \text { a.e. } t \in \bar{I} .
$$

In addition, we define the operator $\Lambda: C(\bar{I} ; Q) \rightarrow C(\bar{I} ; Q)$ by

$$
\Lambda \eta=\int_{-r}^{0} h\left(t+\theta, \varepsilon\left(\tilde{u}_{\eta}\right)\right) \mu(d \theta), \quad \forall \eta \in C(\bar{I} ; Q), \text { a.e. } t \in \bar{I}
$$


Lemma 3.4. The operator $\Lambda$ has a unique fixed point $\eta^{*} \in C(\bar{I} ; Q)$.

Proof. For any $\eta_{1}, \eta_{2} \in C(\bar{I} ; Q)$, let $u_{i}=u_{\eta_{i}}, \tilde{u}_{i}=\tilde{u}_{\eta_{i}}$ and $w_{i}=w_{\eta_{i}}$ with $i=1$, 2. Using (3.22) and arguments similar to those used in the proof of Lemma 3.3, we obtain

$$
\left\|w_{1}(t)-w_{2}(t)\right\|_{V} \leq c_{2}\left(\left\|\eta_{1}(t)-\eta_{2}(t)\right\|_{Q}+\int_{0}^{t}\left\|w_{1}(s)-w_{2}(s)\right\|_{V} d s\right), \quad \text { a.e. } t \in \bar{I},
$$

where

$$
c_{2}=\max \left\{\frac{1}{M}, \frac{c}{M}\right\}
$$

An application of the Gronwall inequality yields

$$
\left\|w_{1}(t)-w_{2}(t)\right\|_{V} \leq c_{3}\left(\left\|\eta_{1}(t)-\eta_{2}(t)\right\|_{Q}+\int_{0}^{t}\left\|\eta_{1}(s)-\eta_{2}(s)\right\|_{Q} d s\right), \quad \text { a.e. } t \in \bar{I},
$$

where

$$
c_{3}=\max \left\{c_{2}, c_{2}^{2} e^{c_{2} T}\right\}
$$

Thus,

$$
\int_{0}^{t}\left\|w_{1}(s)-w_{2}(s)\right\|_{V} d s \leq c_{4} \int_{0}^{t}\left\|\eta_{1}(s)-\eta_{2}(s)\right\|_{Q} d s, \quad \text { a.e. } t \in \bar{I}
$$

where $c_{4}=c_{3}(1+T)$. For the operator $\Lambda$ defined in (3.24), by $\tilde{u} \in \mathfrak{R}(u),(2.5)$, Remark 2.4, and $\mathrm{H}(h)$, we obtain

$$
\begin{aligned}
\left\|\Lambda \eta_{1}(t)-\Lambda \eta_{2}(t)\right\|_{Q} & =\left\|\int_{-r}^{0}\left[h\left(t+\theta, \varepsilon\left(\tilde{u}_{1}\right)\right)-h\left(t+\theta, \varepsilon\left(\tilde{u}_{2}\right)\right)\right] \mu(d \theta)\right\|_{Q} \\
& \leq|\mu|([-r, 0])\left\|h\left(t+\theta, \varepsilon\left(\tilde{u}_{1}\right)\right)-h\left(t+\theta, \varepsilon\left(\tilde{u}_{2}\right)\right)\right\|_{Q} \\
& \leq L_{3}|\mu|([-r, 0])\left\|u_{1}-u_{2}\right\|_{V} \\
& \leq c_{5} \int_{0}^{t}\left\|\eta_{1}-\eta_{2}\right\|_{V} \text { ds, a.e. } t \in \bar{I},
\end{aligned}
$$

where $c_{5}=L_{3}|\mu|([-r, 0]) c_{4}$. By using (3.30) and the similar proof of Lemma 3.3, we get the result of Lemma 3.4. This completes the proof. 
Now we prove Theorem 3.1.

Proof of Theorem 3.1. Let $\eta^{*} \in C(\bar{I} ; Q)$ be the fixed point of $\Lambda$ and let $u_{\eta^{*}} \in C^{1}(\bar{I} ; Q)$ be the function defined by (3.24) for $\eta=\eta^{*}$. For any $v \in V_{1}$ and a.e. $t \in \bar{I}$, it follows from $\dot{u}_{\eta^{*}}=w_{\eta^{*}}$ and (3.22) that

$$
\begin{aligned}
& \left(\mathcal{A}\left(t, \varepsilon\left(\dot{u}_{\eta^{*}}(t)\right)\right), \varepsilon\left(v-\dot{u}_{\eta^{*}}(t)\right)\right)_{Q}+\left(\eta^{*}, \varepsilon\left(v-\dot{u}_{\eta^{*}}(t)\right)\right)_{Q}+j\left(t, S_{t}\left(\dot{u}_{\eta^{*}}\right) ; v\right)-j\left(t, S_{t}\left(\dot{u}_{\eta^{*}}\right) ; \dot{u}_{\eta^{*}}(t)\right) \\
& \quad \geq\left(f(t), v-\dot{u}_{\eta^{*}}(t)\right)_{V} .
\end{aligned}
$$

Now inequality (2.34) follows from (3.24) and (3.30). Moreover, since (3.23) implies $u_{\eta^{*}}(0)=$ $u_{0}$, we conclude that $u_{\eta^{*}}$ is a solution of Problem 2 .

Let $u_{1}, u_{2} \in C\left(\bar{I} ; V_{1}\right)$ be two solutions to Problem 2 and let $w_{i}=\dot{u}_{i}$ for $i=1,2$. Then we have

$$
u_{i}(t)=\int_{0}^{t} w_{i}(s) d s+u_{0}, \quad \text { a.e. } t \in \bar{I} .
$$

For a.e. $t \in \bar{I}$, by the similar argument used in obtaining (3.6), we have

$$
\begin{aligned}
& \left(\mathcal{A}\left(t, \varepsilon\left(w_{1}(t)\right)\right)-\mathcal{A}\left(t, \varepsilon\left(w_{2}(t)\right)\right), \varepsilon\left(w_{1}(t)-w_{2}(t)\right)\right)_{Q} \\
& \quad \leq\left(\Lambda \eta_{1}(t)-\Lambda \eta_{2}(t), \varepsilon\left(w_{1}(t)-w_{2}(t)\right)\right)_{Q}+D\left(t, w_{1}, w_{2}\right),
\end{aligned}
$$

where

$$
\begin{aligned}
& D\left(t, w_{1}, w_{2}\right) \\
& \quad=j\left(t, S_{t}\left(w_{1}\right) ; w_{2}(t)\right)-j\left(t, S_{t}\left(w_{1}\right) ; w_{1}(t)\right)+j\left(t, S_{t}\left(w_{2}\right) ; w_{1}(t)\right)-j\left(t, S_{t}\left(w_{2}\right) ; w_{2}(t)\right) .
\end{aligned}
$$

Using (2.29), (3.8), (2.9), and $\mathrm{H}(g)$, we deduce that

$$
D\left(t, w_{1}, w_{2}\right) \leq c_{1} \int_{0}^{t}\left\|w_{1}(s)-w_{2}(s)\right\|_{V} d s\left\|w_{1}(t)-w_{2}(t)\right\|_{V} \text {, a.e. } t \in \bar{I} .
$$

From the assumption $\mathrm{H}(\mathcal{A})$ and relations (3.30)-(3.35), we know that, for a.e. $t \in \bar{I}$,

$$
\left\|w_{1}(t)-w_{2}(t)\right\|_{V} \leq c_{6}\left(\left\|u_{1}(t)-u_{2}(t)\right\|_{V}+\int_{0}^{t}\left\|w_{1}(s)-w_{2}(s)\right\|_{V} d s\right),
$$

where

$$
c_{6}=\max \left\{\frac{L_{3}|\mu|([-r, 0])}{M}, \frac{c_{1}}{M}\right\} .
$$


An application of the Gronwall inequality yields

$$
\left\|w_{1}(t)-w_{2}(t)\right\|_{V} \leq c_{7}\left(\left\|u_{1}(t)-u_{2}(t)\right\|_{V}+\int_{0}^{t}\left\|u_{1}(s)-u_{2}(s)\right\|_{V} d s\right), \quad \text { a.e. } t \in \bar{I},
$$

where

$$
c_{7}=\max \left\{c_{6}, c_{6}^{2} e^{c_{6} T}\right\}
$$

Recalling the definition (3.32) of $u_{1}$ and $u_{2}$, and letting $c_{8}=c_{7}(1+T)$, we obtain

$$
\left\|w_{1}(t)-w_{2}(t)\right\|_{V} \leq c_{8} \int_{0}^{t}\left\|w_{1}(s)-w_{2}(s)\right\|_{V} d s, \quad \text { a.e. } t \in \bar{I}
$$

and so the Gronwall inequality implies that $w_{1}=w_{2}$. By definition (3.32), we see that $u_{1}=u_{2}$, which completes the proof of Theorem 3.1.

Theorem 3.5. Problem 1 has a unique solution $u \in C^{1}\left(\bar{I} ; V_{1}\right)$.

Proof. Let $\zeta \in C\left(\bar{I} ; V_{1}\right)$ and denote by $u_{\zeta} \in C\left(\bar{I} ; V_{1}\right)$ the solution of the following problem:

$$
\begin{aligned}
(\mathcal{A}(t, \varepsilon & \left.\left.\left(\dot{u}_{\zeta}(t)\right)\right), \varepsilon\left(v-\dot{u}_{\zeta}(t)\right)\right)_{Q}+\left(\mathcal{G} h\left(t, \varepsilon\left(\tilde{u}_{\zeta}\right)\right), \varepsilon\left(v-\dot{u}_{\zeta}(t)\right)\right)_{Q} \\
& +j\left(t, S_{t}\left(\dot{u}_{\zeta}\right) ; v\right)-j\left(t, S_{t}\left(\dot{u}_{\zeta}\right) ; \dot{u}_{\zeta}(t)\right) \\
\geq & \left(f(t)-\zeta(t), v-\dot{u}_{\zeta}(t)\right)_{V}, \quad \forall v, \tilde{u}_{\zeta} \in V_{1}, \text { a.e. } t \in \bar{I},
\end{aligned}
$$

where

$$
\left(ß\left(t, \varepsilon\left(u_{\zeta}(t)\right)\right), \varepsilon\left(v-\dot{u}_{\zeta}(t)\right)_{Q}=\left(\zeta(t), v-\dot{u}_{\zeta}(t)\right)_{V} .\right.
$$

From Theorem 3.1, we know that there exists a unique solution $u_{\zeta}$ for problem (3.41).

Consider the operator $\Upsilon: C\left(\bar{I} ; V^{*}\right) \rightarrow C\left(\bar{I} ; V^{*}\right)$ defined by

$$
\Upsilon \zeta(t)=B\left(t, \varepsilon\left(u_{\zeta}(t)\right)\right), \quad \forall \zeta \in C\left(\bar{I} ; V_{1}\right) \text {, a.e. } t \in \bar{I} .
$$

Now we show that the operator $\Upsilon$ has a unique fixed point. In fact, for any $\zeta_{1}, \zeta_{2} \in C\left(\bar{I} ; V_{1}\right)$, let $u_{1}=u_{\zeta_{1}}$ and $u_{2}=u_{\zeta_{2}}$ be the corresponding solutions to (3.41). Then it is easy to see 
that $u_{1}, u_{2} \in C\left(\bar{I} ; V_{1}\right)$. For any $\tilde{u}_{1} \in \mathfrak{R}\left(u_{1}\right)$ and $\tilde{u}_{2} \in \mathfrak{R}\left(u_{2}\right)$, by the similar argument used in obtaining (3.33), we have

$$
\begin{aligned}
\left(\zeta_{2}(t)-\right. & \left.\zeta_{1}(t), w_{1}(t)-w_{2}(t)\right)_{V}+D\left(t, w_{1}, w_{2}\right) \\
\geq & \left(\mathcal{A}\left(t, \varepsilon\left(w_{1}(t)\right)\right)-\mathcal{A}\left(t, \varepsilon\left(w_{2}(t)\right)\right), \varepsilon\left(w_{1}(t)-w_{2}(t)\right)\right)_{Q} \\
& +\left(\mathcal{G} h\left(t, \varepsilon\left(\tilde{u}_{1}\right)\right)-\mathcal{G} h\left(t, \varepsilon\left(\tilde{u}_{2}\right)\right), \varepsilon\left(w_{1}(t)-w_{2}(t)\right)\right)_{Q}, \quad \text { a.e. } t \in \bar{I} .
\end{aligned}
$$

By $\mathrm{H}(\mathcal{A}), \mathrm{H}(h),(2.5),(3.30),(3.32)$, and (3.35), we get

$$
\left\|w_{1}(t)-w_{2}(t)\right\|_{V} \leq c_{9}\left(\left\|\zeta_{1}(t)-\zeta_{2}(t)\right\|_{V}+\int_{0}^{t}\left\|w_{1}(s)-w_{2}(s)\right\|_{V} d s\right), \quad \text { a.e. } t \in \bar{I},
$$

where

$$
c_{9}=\max \left\{\frac{1}{M+M^{\prime}}, \frac{1+c}{M+M^{\prime}}\right\}
$$

An application of the Gronwall inequality yields

$$
\int_{0}^{t}\left\|w_{1}(s)-w_{2}(s)\right\|_{V} d s \leq c_{10} \int_{0}^{t}\left\|\zeta_{1}(s)-\zeta_{2}(s)\right\|_{V} d s, \quad \text { a.e. } t \in \bar{I}
$$

where $c_{10}=(1+T) \max \left\{c_{9}, c_{9}^{2} e^{c_{9} T}\right\}$. From $H(B),(3.43)$, and (3.47), we have

$$
\left\|\Upsilon \zeta_{1}(t)-\Upsilon \zeta_{2}(t)\right\|_{V}^{2} \leq c_{11} \int_{0}^{t}\left\|\zeta_{1}(s)-\zeta_{2}(s)\right\|_{V}^{2} d s, \quad \text { a.e. } t \in \bar{I},
$$

where $c_{11}=T c_{10}^{2} L_{1}^{2}$. Iterating the last inequality $p$ times, we obtain

$$
\left\|\Upsilon^{p} \zeta_{1}(t)-\Upsilon^{p} \zeta_{2}(t)\right\|_{V}^{2} \leq \frac{c_{11}^{p} t^{p-1}}{(p-1) !} \int_{0}^{t}\left\|\zeta_{1}(s)-\zeta_{2}(s)\right\|_{V}^{2} d s, \quad \text { a.e. } t \in \bar{I},
$$

which leads to

$$
\left\|\Upsilon^{p} \zeta_{1}-\Upsilon^{p} \zeta_{2}\right\|_{L^{2}(\bar{I} ; V)} \leq\left(\frac{c_{11}^{p} T^{p}}{(p-1) !}\right)^{1 / 2}\left\|\zeta_{1}-\zeta_{2}\right\|_{L^{2}(\bar{I} ; V) \prime} \quad \text { a.e. } t \in \bar{I}
$$

Since $\lim _{p \rightarrow \infty}\left(c_{11}^{p} T^{p} /(p-1) !\right)^{1 / 2}=0$, the previous inequality implies that, for $p$ large enough, a power $\Upsilon^{p}$ of $\Upsilon$ is a contraction. It follows that there exists a unique element $\zeta^{*} \in V_{1}$ such that $\Upsilon^{p} \zeta^{*}=\zeta^{*}$. Moreover, since

$$
\Upsilon^{p}\left(\Upsilon \zeta^{*}\right)=\Upsilon\left(\Upsilon^{p} \zeta^{*}\right)=\Upsilon \zeta^{*}
$$


we deduce that $\Upsilon \zeta^{*}$ is also a fixed point of the operator $\Upsilon^{p}$. By the uniqueness of the fixed point that $\Upsilon \zeta^{*}=\zeta^{*}$, we know that $\zeta^{*}$ is a fixed point of $\Upsilon$. The uniqueness of the fixed point of $\Upsilon$ results straightforward from the uniqueness of the fixed point of $\Upsilon^{p}$. This implies that $u_{\zeta^{*}}$ is the unique solution of Problem 1, which completes the proof of Theorem 3.5.

Remark 3.6. When $\mathcal{G}=0$ and all the viscosity and elasticity operators $\mathcal{A}$ and $\mathbb{B}$ are explicitly time dependent, Theorem 3.5 reduces to Theorem 10.2 of [1]. Furthermore, Theorem 3.5 is also a generalization of Theorem 2.1 of [24].

\section{A Convergence Result}

In this section, we study the dependence of the solution to Problem 1 with respect to perturbations of the operator $h$. We assume that $\mathrm{H}(\mathcal{A}), \mathrm{H}(\mathbb{B}), \mathrm{H}(g)$, and $\mathrm{H}(h)$ hold and, for any $\beta>0$, let $h_{\beta}$ be a perturbation of the operator $h$.

We consider the following problem.

Problem 4. Find $u_{\beta}: \bar{I} \rightarrow V_{1}$ such that

$$
\begin{gathered}
\left(\mathcal{A}\left(t, \varepsilon\left(\dot{u}_{\beta}(t)\right)\right), \varepsilon\left(v-\dot{u}_{\beta}(t)\right)\right)_{Q}+\left(B\left(t, \varepsilon\left(u_{\beta}(t)\right)\right), \varepsilon\left(v-\dot{u}_{\beta}(t)\right)\right)_{Q} \\
+\left(\mathcal{G} h_{\beta}\left(t, \varepsilon\left(\tilde{u}_{\beta}\right)\right), \varepsilon\left(v-\dot{u}_{\beta}(t)\right)\right)_{Q}+j\left(t, S_{t}\left(\dot{u}_{\beta}\right) ; v\right)-j\left(t, S_{t}\left(\dot{u}_{\beta}\right) ; \dot{u}_{\beta}(t)\right) \\
\geq\left(f(t), v-\dot{u}_{\beta}(t)\right)_{V^{\prime}} \quad \forall v \in V_{1}, \tilde{u}_{\beta} \in \Re\left(u_{\beta}\right), \\
u_{\beta}(0)=u_{0} .
\end{gathered}
$$

It follows from Theorem 3.5 that, for each $\beta>0$, Problem 4 has a unique solution denoted by $u_{\beta} \in C\left(\bar{I} ; V_{1}\right)$.

In order to get the convergence result, we need the following assumption:

$$
\lim _{\beta \rightarrow 0}\left\|h_{\beta}(x, t, \varepsilon)-h(x, t, \varepsilon)\right\|_{Q}=0 .
$$

Now we give the convergence result.

Theorem 4.1. Assume that $H(\mathcal{A}), H(B), H(g), H(h)$, and (2.28) hold. Then the solution $u_{\beta}$ of Problem 4 converges to the solution $u$ of Problem 1, that is,

$$
\lim _{\beta \rightarrow 0}\left\|u_{\beta}-u\right\|_{C\left(\bar{I} ; V_{1}\right)}=0
$$

Proof. For any $\beta>0$ and a.e. $t \in \bar{I}$, let

$$
\begin{gathered}
\eta_{\beta}=\int_{-r}^{0} h_{\beta}\left(t+\theta, \varepsilon\left(\tilde{u}_{\beta}(\theta)\right)\right) \mu(d \theta), \\
\eta=\int_{-r}^{0} h(t+\theta, \varepsilon(\tilde{u}(\theta))) \mu(d \theta) .
\end{gathered}
$$


Keeping in mind (4.1) and (2.33), and using $\mathrm{H}(\mathcal{A})$ and $\mathrm{H}(\mathbb{B})$, by the similar argument used in obtaining (3.36), we have

$$
\left\|w_{\beta}(t)-w(t)\right\|_{V} \leq c_{12}\left(\left\|\eta_{\beta}(t)-\eta(t)\right\|_{V}+\int_{0}^{t}\left\|w_{\beta}(s)-w(s)\right\|_{V} d s\right), \quad \text { a.e.t } \in \bar{I},
$$

where

$$
c_{12}=\max \left\{\frac{1}{M}, \frac{c+L_{1}}{M}\right\}
$$

Hence,

$$
\int_{0}^{t}\left\|w_{\beta}(s)-w(s)\right\|_{V} d s \leq c_{13} \int_{0}^{t}\left\|\eta_{\beta}(s)-\eta(s)\right\|_{V} d s, \quad \text { a.e.t } \in \bar{I},
$$

where

$$
c_{13}=(1+T) \max \left\{c_{12}, c_{12}^{2} e^{c_{12} T}\right\}
$$

For a.e. $t \in \bar{I}$, it follows from (4.5), (2.5), and Remark 2.4 that

$$
\begin{aligned}
\left\|\eta_{\beta}(t)-\eta(t)\right\|_{Q} \leq & |\mu|([-r, 0])\left\|h_{\beta}\left(t+\theta, \varepsilon\left(\tilde{u}_{\beta}(\theta)\right)\right)-h(t+\theta, \varepsilon(\tilde{u}(\theta)))\right\|_{Q} \\
\leq & |\mu|([-r, 0])\left[\left\|h_{\beta}\left(t+\theta, \varepsilon\left(\tilde{u}_{\beta}(\theta)\right)\right)-h_{\beta}(t+\theta, \varepsilon(\tilde{u}(\theta)))\right\|_{Q}\right. \\
& \left.+\left\|h_{\beta}(t+\theta, \varepsilon(\tilde{u}(\theta)))-h(t+\theta, \varepsilon(\tilde{u}(\theta)))\right\|_{Q}\right] .
\end{aligned}
$$

Noting $\tilde{u}_{\beta} \in \mathfrak{R}\left(u_{\beta}\right)$, from the last inequality, (4.3), (4.8), and $\mathrm{H}(h)$, we have

$$
\left\|w_{\beta}(t)-w(t)\right\|_{V} \leq c_{13}|\mu|([-r, 0]) T L_{3} \int_{0}^{t}\left\|w_{\beta}(s)-w(s)\right\|_{V} d s, \quad \text { a.e.t } \in \bar{I} .
$$

It follows from the Gronwall inequality that $w_{\beta}=w$ and so the convergence result (4.4) is a consequence of (3.32). This completes the proof.

\section{Acknowledgments}

The authors are grateful to the editor and the referees for their valuable comments and suggestions. This work was supported by the Key Program of NSFC (Grant no. 70831005), the National Natural Science Foundation of China (11171237, 11101069), and the Scientific Research Foundation of Yunnan Provincial Education Office (2010Z024). 


\section{References}

[1] W. Han and M. Sofonea, Quasistatic Contact Problems in Viscoelasticity and Viscoplasticity, vol. 30 of Studies in Advanced Mathematics, American Mathematical Society/ International Press, Providence, RI, USA, 2002.

[2] X. W. Chen, X. L. Han, and F. Luo S Wu, "Fiber element based elastic-plastic analysis procedure and engineering application," Procedia Engineering, vol. 14, pp. 1807-1815, 2011.

[3] O. Markus and P. Terhi, "Computational framework for common visco-elastic models in engineering based on the theory of rheology," Computers and Geotechnics, vol. 42, pp. 145-156, 2012.

[4] P. R. Marur, "An engineering approach for evaluating effective elastic moduli of particulate composites," Materials Letters, vol. 58, no. 30, pp. 3971-3975, 2004.

[5] I. Argatov, "Sinusoidally-driven flat-ended indentation of time-dependent materials: asymptotic models for low and high rate loading," Mechanics of Materials, vol. 48, pp. 56-70, 2012.

[6] F. Maceri, M. Marino, and G. Vairo, "An insight on multiscale tendon modeling in muscle-tendon integrated behavior," Biomechanics and Modeling in Mechanobiology, vol. 11, no. 3-4, pp. 505-517, 2012.

[7] D. Duvaut and J. L. Lions, Inequalities in Mechanics and Physics, Springer, Berlin, Germany, 1976.

[8] N. Kikuchi and J. T. Oden, Contact Problems in Elasticity: A Study of Variational Inequalities and Finite Element Methods, vol. 8, SIAM, Philadelphia, Pa, USA, 1988.

[9] O. Chau, J. R. Fernández, M. Shillor, and M. Sofonea, "Variational and numerical analysis of a quasistatic viscoelastic contact problem with adhesion," Journal of Computational and Applied Mathematics, vol. 159, no. 2, pp. 431-465, 2003.

[10] A. Rodriguez-Aros, M. Sofonea, and J. Viano, "A class of evolutionary variational inequalities with volterratype integral term," Mathematical Models and Methods in Applied Sciences, vol. 14, pp. 555-577, 2004.

[11] M. Shillor, "Special issue on recent advances in contact mechanics," Mathematical and Computer Modelling, vol. 28, pp. 4-8, 1998.

[12] W. Han and M. Sofonea, "Evolutionary variational inequalities arising in viscoelastic contact problems," SIAM Journal on Numerical Analysis, vol. 38, no. 2, pp. 556-579, 2000.

[13] O. Chau, D. Motreanu, and M. Sofonea, "Quasistatic frictional problems for elastic and viscoelastic materials," Applications of Mathematics, vol. 47, no. 4, pp. 341-360, 2002.

[14] M. Delost and C. Fabre, "On abstract variational inequalities in viscoplasticity with frictional contact," Journal of Optimization Theory and Applications, vol. 133, no. 2, pp. 131-150, 2007.

[15] E. A. H. Vollebregt and H. M. Schuttelaars, "Quasi-static analysis of two-dimensional rolling contact with slip-velocity dependent friction," Journal of Sound and Vibration, vol. 331, pp. 2141-2155, 2012.

[16] M. Shillor, M. Sofonea, and J. J. Telega, Models and Analysis of Quasistatic Contact, vol. 655 of Lecture Notes in Physics, Springer, Berlin, Germany, 2004.

[17] S. Migórski, A. Ochal, and M. Sofonea, "An evolution problem in nonsmooth elasto-viscoplasicity," Nonlinear Analysis: Theory, Methods \& Applications, vol. 71, no. 12, pp. e2766-e2771, 2009.

[18] S. Migórski, A. Ochal, and M. Sofonea, "History-dependent subdifferential inclusions and hemivariational inequalities in contact mechanics," Nonlinear Analysis: Real World Applications, vol. 12, no. 6, pp. 3384-3396, 2011.

[19] N. Costea and A. Matei, "Contact models leading to variational-hemivariational inequalities," Journal of Mathematical Analysis and Applications, vol. 386, no. 2, pp. 647-660, 2012.

[20] V. Comincioli, "A result concerning a variational inequality of evolution for operators of first order in t with retarded terms," Annali di Matematica Pura ed Applicata, vol. 88, pp. 357-378, 1971.

[21] J. Y. Park, J. U. Jeong, and Y. H. Kang, "Optimal control of parabolic variational inequalities with delays and state constraint," Nonlinear Analysis: Theory, Methods E Applications, vol. 71, no. 12, pp. e329-e339, 2009.

[22] S. W. Zhu, "Optimal control of variational inequalities with delays in the highest order spatial derivatives," Acta Mathematica Sinica, vol. 22, no. 2, pp. 607-624, 2006.

[23] J. M. Yong and L. P. Pan, "Quasi-linear parabolic partial differential equations with delays in the highest order spatial derivatives," Australian Mathematical Society, vol. 54, no. 2, pp. 174-203, 1993.

[24] M. Sofonea, A. Rodríguez-Arós, and J. M. Viaño, "A class of integro-differential variational inequalities with applications to viscoelastic contact," Mathematical and Computer Modelling, vol. 41, no. 11-12, pp. 1355-1369, 2005.

[25] M. Campo, J. R. Fernández, and Á. Rodríguez-Arós, “A quasistatic contact problem with normal compliance and damage involving viscoelastic materials with long memory," Applied Numerical Mathematics, vol. 58, no. 9, pp. 1274-1290, 2008. 
[26] I. Figueiredo and L. Trabucho, "A class of contact and friction dynamic problems in thermoelasticity and in thermoviscoelasticity," International Journal of Engineering Science, vol. 33, no. 1, pp. 45-66, 1995.

[27] A. Kulig and S. Migórski, "Solvability and continuous dependence results for second order nonlinear evolution inclusions with a Volterra-type operator," Nonlinear Analysis: Theory, Methods E Applications, vol. 75, no. 13, pp. 4729-4746, 2012.

[28] A. Rodriguez-Aros, M. Sofonea, and J. Viano, "Numerical analysis of a frictional contact problem for viscoelastic materials with long-term memory," Advances in Mechanics and Mathematics, vol. 18, pp. 1-11, 2009. 


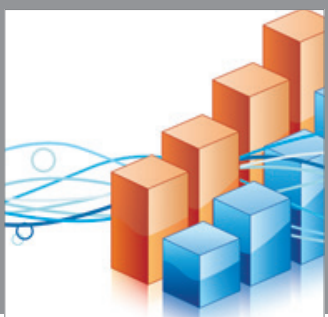

Advances in

Operations Research

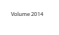

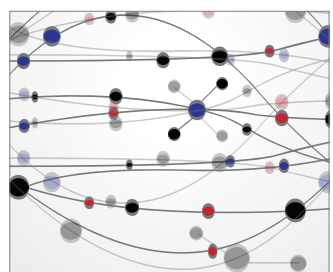

\section{The Scientific} World Journal
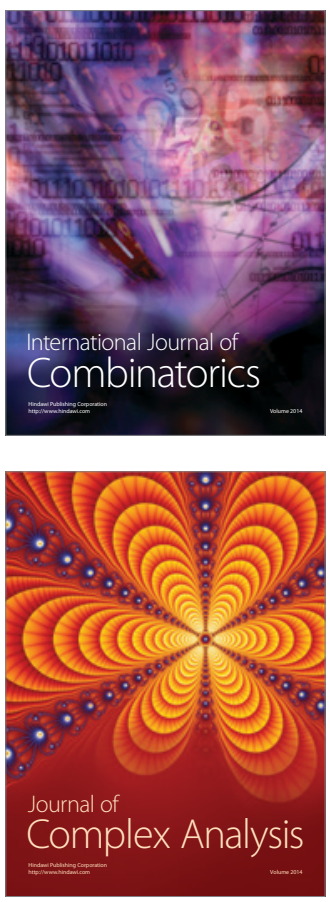

International Journal of

Mathematics and

Mathematical

Sciences
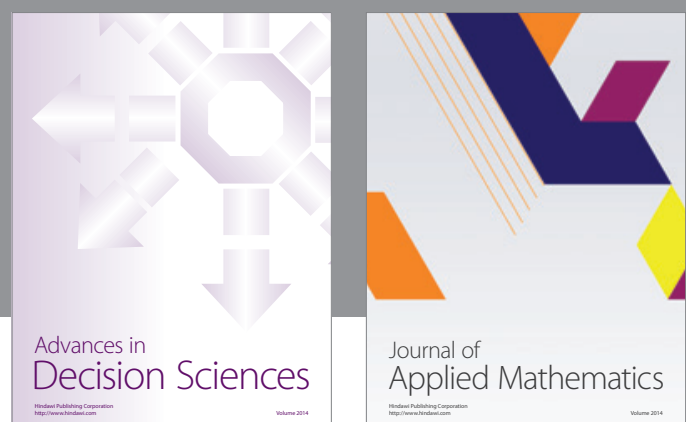

Journal of

Applied Mathematics
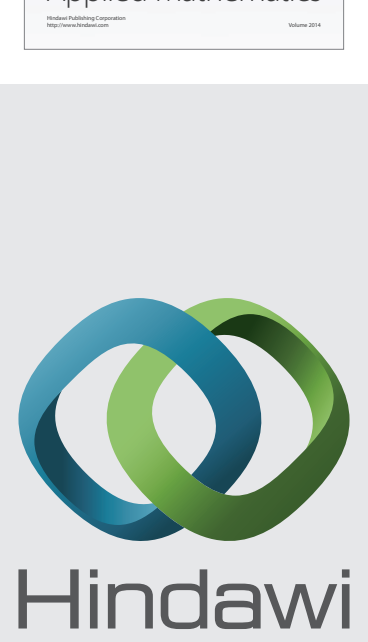

Submit your manuscripts at http://www.hindawi.com
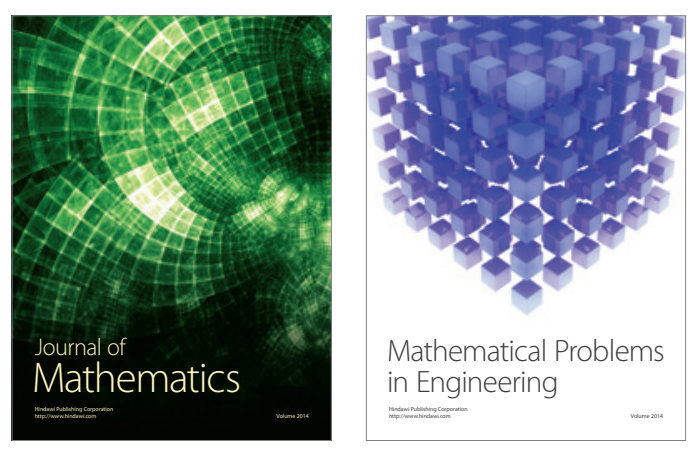

Mathematical Problems in Engineering
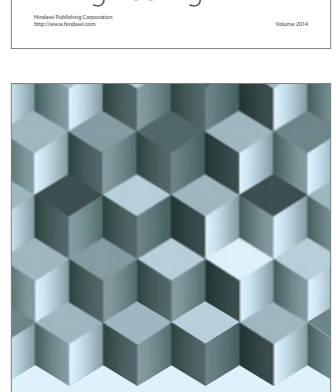

Journal of

Function Spaces
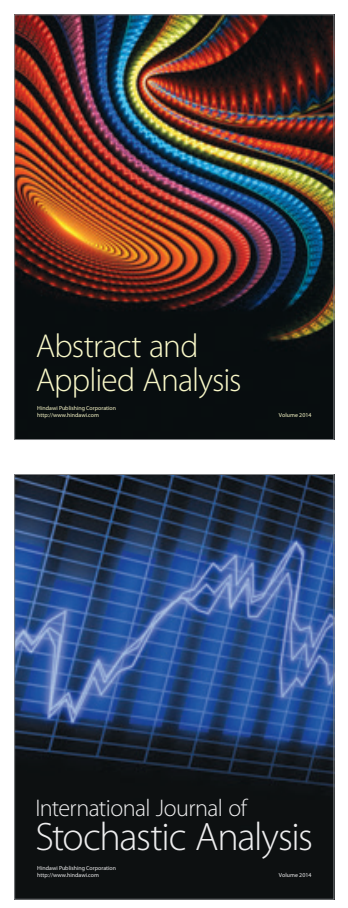

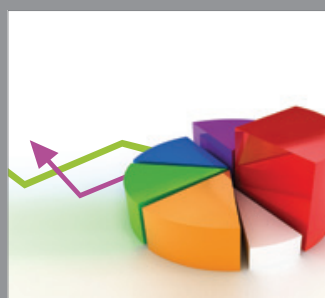

ournal of

Probability and Statistics

Promensencen
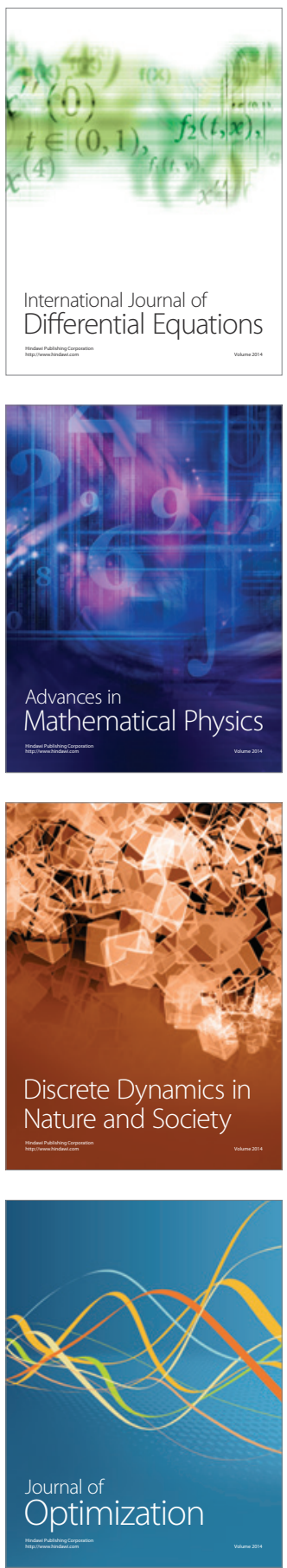